treatments with abatacept, tocilizumab, rituximab or ustekinumab were recorded.

Average baseline BASDAI was 6.54 (0.4-12.8), and 5.01 (0-10) after 6 mo SCK therapy. Baseline BASFI was $5.8(0.4-10)$ and $4.8(0-9.6)$ at 6 months. Adverse events did not differ from those reported in clinical trials.

Conclusion: Population receiving SCK in real-world setting is different to clinical trials: more complex patientes, with higher disease activity, higher use of previous bDMARDs and higher comorbidity, particularly cardiovascular risk, infections or malignancy that could preclude the use of other biologic therapies. A relevant percentage of off-label prescription in $\mathrm{nrAx}$ $\mathrm{SpA}$ is reported. Further studys are needed to characterize this population and its clinical response and tolerability to SCK therapy.

Disclosure of Interests: Valentina Emperiale: None declared, Jose Campos Esteban: None declared, Carolina Merino Argumánez: None declared, Javier Bachiller-Corral: None declared, Marta Valero: None declared, Beatriz Joven-Ibáñez Speakers bureau: Celgene, Novartis, MSD, Pfizer, AbbVie, and Janssen, María Martín: None declared, Ana Pérez Gómez: None declared

DOI: 10.1136/annrheumdis-2019-eular.284

\section{AB0702 $\quad$ TREATMENT EFFECT OF TUMOR NECROSIS FACTOR A INHIBITORS ON MAGNETIC RESONANCE IMAGING PROGRESSION IN PATIENTS WITH SPONDYLOARTHRITIS: A META-ANALYSIS}

Yupeng Huang, Yuehong Chen, Tao Liu, Sang Lin, Geng Yin, Qibing Xie. West China Hospital, Sichuan University, Chengdu, China

Background: Treatment effect of tumor necrosis factor $\alpha$ inhibitors (TNFi) in patients with spondyloarthritis ( $\mathrm{SpA}$ ) had been proved by plenty of studies that could remarkably eliminate symptoms, signs, and laboratory inflammatory manifestations ${ }^{[1,2]}$. Simultaneously, the treatment effect of $\mathrm{TNFi}$ on delaying radiographic progression in $\mathrm{SpA}$ was often reported ${ }^{[3,4]}$, including magnetic resonance imaging (MRI) which has a high sensitivity on detecting the earliest inflammation [5]

Objectives: A meta-analysis was performed to summarize the treatment effect of TNFi on MRI progression reflected by SPARCC score in SpA patients

Methods: Comprehensive search was conducted in the electronic databases of OVID Medline, OVID EMBASE and Cochrane library on Nov.27, 2018. All randomized controlled trials (RCTs) focused on MRI progression and disease activity in SpA patients treated with TNFi were included. Primary outcome was the Spondyloarthritis Research Consortium Canad (SPARCC) MRI scoring system, accompanied with or without other outcomes, including Ankylosing Spondylitis Disease Activity Score (ASDAS), Bath Ankylosing Spondylitis disease activity index (BASDAI), Bath Ankylosing Spondylitis functional index (BASFI), C-reactive protein (CRP). Data were pooled by mean differences (MD) with $95 \%$ confidence intervals $(\mathrm{Cl})$ and publication bias was assessed by funnel plot. Sensitivity analysis was performed to test the result robustness. Jadad scale was applied to assess the methodology quality of included trials. Two reviewers independently selected studies, extracted data, and assessed study quality.

Results: Totally 10 RCTs enrolled 1006 patients with high methodology were included. Compared with control group, TNFi significantly improved SPARCC score at sacroiliac joint (SIJ) $(M D=2.84,95 \% \mathrm{Cl}$ 2.43-3.25), SPARCC score of spine (MD=1.87, 95\% Cl 1.27-2.46), ASDAS $(\mathrm{MD}=0.96,95 \% \mathrm{Cl} 0.71-1.20)$, BASDAl $(\mathrm{MD}=1.15,95 \% \mathrm{Cl} 0.51-1.78)$, BASFI (MD $=0.95,95 \% \mathrm{Cl} 0.51-1.40)$, and CRP (MD=4.64, 95\% Cl 1.068.23 in double-blind phase. Subgroup analyses by disease subgroup and individual TNFi showed treatment effect of TNFi on delaying MRI progression was not affected by disease subgroup and individual TNFi. Sensitivity analysis showed the treatment effects of TNFi versus placebo were consistent with TNFi versus controls, suggesting the results of the study were robust. The funnel plot of SPARCC score at SIJ based on TNFi versus control in double-blind phase was asymmetric, suggesting there might have potential publication bias.

Conclusion: TNFi are effective to treat SpA patients and delay MRI progression which are assessed by SPARCC score and treatment effect is consistent among disease subgroups and individual TNFi.

\section{REFERENCES}

[1] Baraliakos X, Listing J, Fritz C, et al. Persistent clinical efficacy and safety of infliximab in ankylosing spondylitis after 8 years-early clinical response predicts long-term outcome. Rheumatology (Oxford). 2011; 50(9):1690-9.
[2] Sfikakis PP. The first decade of biologic TNF antagonists in clinical practice: lessons learned, unresolved issues and future directions. Curr Dir Autoimmun. 2010;11:180-210.

[3] Wanders A, van der Heijde D, Landewe R, et al. Nonsteroidal antiinflammatory drugs reduce radiographic progression in patients with ankylosing spondylitis: a randomized clinical trial. Arthritis Rheum. 2005;52(6):175665.

[4] Poddubnyy D, Rudwaleit M, Haibel H, et al.Effect of nonsteroidal antiinflammatory drugs on radiographic spinal progression in patients with axia spondyloarthritis: results from the German Spondyloarthritis Inception Cohort. Ann Rheum Dis. 2012;71:1616-22.

[5] Puhakka KB, Jurik AG, Schiottz-Christensen B, et al. Magnetic resonance imaging of sacroiliitis in early seronegative spondylarthropathy. Abnormalities correlated to clinical and laboratory findings. Rheumatology (Oxford) 2004;43(2):234-7.

Disclosure of Interests: None declared

DOI: 10.1136/annrheumdis-2019-eular.2541

\section{AB0703 INFLAMMATORY BOWEL DISEASES AMONG SECUKINUMAB-TREATED PATIENTS: FIRST RESULTS OF THE MISSIL REGISTRY}

Jean-Guillaume Letarouilly ${ }^{1}$, Benjamin Pariente ${ }^{1}$, Philippe Goupille ${ }^{2}$

Pascal Claudepierre ${ }^{3}$, Stephane Varin ${ }^{4}$, Sylvain Lanot ${ }^{5}$, Emmanuelle Dernis ${ }^{6}$, Loïc Le Dantec ${ }^{7}$, Philippe Gaudin ${ }^{8}$, Denis Jullien ${ }^{9}$, Thao Pham ${ }^{10}$, Rene-Marc Flipo ${ }^{1}$ ${ }^{1}$ Lille University, Lille, France; ${ }^{2}$ Tours University Hospital, Rheumatology, Tours, France; ${ }^{3}$ Henri Mondor University Hospital, Rheumatology, Creteil, France; ${ }^{4}$ Vendee Hospital, Rheumatology, La Roche sur Yon, France; ${ }^{5}$ Inter-Hospital, Alençon, France; ${ }^{6}$ Le Mans Hospital, Rheumatology, Le Mans, France; ${ }^{7}$ HéninBeaumont Hospital, Rheumatology, Hénin-Beaumont, France; ${ }^{8}$ Grenoble University Hospital, Rheumatology, Grenoble, France; ${ }^{9}$ Lyon University Hospital, Dermatology, Lyon, France; ${ }^{10}$ Aix Marseille Univ, APHM, Marseille, France

Background: Interleukin 17 (IL17) blockade is a therapeutic alternative for patients who are Tumor Necrosis Factor inhibitor-Inadequate Responders However, in randomized controlled trials, cases of de novo inflammatory bowel diseases (IBD) have been reported in patients treated by IL17 inhibitors.

Objectives: To describe patients treated by $\mathrm{IL} 17$ inhibitors developing new onset IBD (Crohn's disease and ulcerative colitis).

Methods: A French national registry called MISSIL was started in Febru ary 2018 to collect the cases of these patients. This registry is con ducted by rheumatologist, dermatologist and gastroenterologist learned societies specialized on immune-mediated inflammatory diseases (IMID). In France, secukinumab has been granted market authorization in June 2016 and ixekizumab in April 2018.

Results: 12 cases were reported between February and December 2018 11 patients with new onset Crohn's disease and one with ulcerative colitis. Mean age was $51 \pm 14.3$ years old and 8/12 were female. 6 pre sented an axial spondyloarthritis, 3 a peripheral spondyloarthritis and 3 both. 9/10 were HLA-B27 positive. 4/10 have a radiographic sacroiliitis and $3 / 10$ a MRI sacroiliitis. Only one was biological Disease-modifying antirheumatic drug (bDMARD)-naïve. Crohn's disease was mainly located at the ileum, colon and rectum. The mean time to onset of symptoms was $4.9 \pm 5.6$ months. The main symptoms were diarrhoea, nausea and vomiting and loss of weight. Median CRP at the onset of symptoms was $107 \mathrm{mg} / \mathrm{L}$ (66.5-200.7). 9 patients underwent biopsies. 5 were in favour of Crohn's disease. IL17 inhibitors were consistently stopped. Patients were treated by corticosteroids (9/12), mesalazine (3/12), methotrexate (2/ $12)$, thiopurines (1/12), infliximab (3/12), adalimumab (1/12), golimumab (1/ 12), ustekinumab (3/12). The evolution was favourable under treatmen with healing (5/12), improvement $(4 / 12)$ or stabilization (1/12). One patient worsened under treatment and one died (massive myocardial infarction).

Conclusion: IBD under IL 17 inhibitors are rare and lead to discuss the potential iatrogenic role of IL 17 inhibitor drugs. Further cases and case control studies are needed to better characterize this complication and identify patients at risk to develop IBD under IL 17 inhibitor.

Disclosure of Interests: Jean-Guillaume Letarouilly: None declared, Benjamin Pariente: None declared, Philippe Goupille Grant/research support from: Financial compensation received from MSD on a pro-rota basis for participation in Scientific Committee meetings and functions for this study, Speakers bureau: Abbvie, Biogaran, BMS, Hospira, Janssen, MSD, Pfizer, Sanofi-Genzyme, UCB, Pascal Claudepierre Consultant for: Honoraria from Novartis as steering committe of this survey, Stephane Varin: None declared, Sylvain Lanot: None declared, Emmanuelle Dernis: None declared, Loïc Le Dantec: None declared, Philippe Gaudin Speakers 
bureau: Roche, Chugai, BMS, Abbvie, Servier, Pfizer, MSD, UCB, ESAOTE, Genévrier, Janssen, Novartis, Lilly, Biogen, Amge, Denis Jullien Consultant for: Abbott, Celgene, Eli Lilly, Janssen, MSD, Novartis, Pfizer, Serono UCB, Fresenius-Kabi, Biogen, Leo-Pharma and Sandoz, Speakers bureau: Abbott, Celgene, Eli Lilly, Janssen, MSD, Novartis, Pfizer, LeoPharma, Serono, Thao Pham Speakers bureau: Lilly, Novartis, Rene-Marc Flipo Consultant for: Advisory board: Bristol-Myers Squibb DOI: 10.1136/annrheumdis-2019-eular.2573

\section{AB0704 EFFECTIVENESS AND SAFETY OF INFLIXIMAB AND GOLIMUMAB IN ANKYLOSING SPONDYLITIS PATIENTS FROM A PROSPECTIVE OBSERVATIONAL REGISTRY}

Proton Rahman ${ }^{1}$, Derek Haaland ${ }^{2}$, Dalton Sholter ${ }^{3}$, Michael Starr ${ }^{4}$, Arthur Karasik ${ }^{5}$, Michelle Teo ${ }^{6}$, Sanjay Dixit ${ }^{7}$, Ariel Masetto ${ }^{8}$, Anna Jarosynska ${ }^{9}$, Pauline Boulos ${ }^{7}$, Maqbool Sheriff ${ }^{10}$, Jacqueline Stewart ${ }^{11}$, Emmanouil Rampakakis ${ }^{12}$, Odalis Asin Miilan ${ }^{13}$, Allen Lehman ${ }^{13}$, Meagan Rachich ${ }^{14}$, Francois Nantel $\left.\right|^{13} .{ }^{1}$ Memorial University of Newfoundland, St. John's, Canada; ${ }^{2}$ The Waterside Clinic, Barrie, Canada; ${ }^{3}$ University of Alberta, Edmonton, Canada; ${ }^{4}$ McGill University Health Centre, Montréal, Canada; ${ }^{5}$ Ontario Rheumatology Association, Aurora, Canada; ${ }^{6}$ Balfour Medical Clinic, Penticton, Canada; ${ }^{7}$ McMaster University, Hamilton, Canada; ${ }^{8}$ Université de Sherbrooke, Sherbrooke, Canada; ${ }^{9}$ Private Clinic, Burlington, Canada: ${ }^{10}$ Nanaimo Regional General Hospital, Nanaimo, Canada: ${ }^{11}$ Penticton Regional Hospital, Penticton, Canada; ${ }^{12}$ JSS Medical Research, Montreal, Canada; ${ }^{13}$ Janssen Inc, Toronto, Canada; ${ }^{14}$ Janssen Inc., Toronto, Canada

Background: Long-term registries are essential to evaluate new therapies in a patient population that differs from clinical trial and usually varies over time.

Objectives: To describe the profile of ankylosing spondylitis (AS) patients treated with infliximab (IFX) or golimumab (GLM) treatment in Canadian routine care along with its long-term effectiveness and safety.

Methods: 810 AS patients treated with IFX or GLM were enrolled into the Biologic Treatment Registry Across Canada (BioTRAC) registry between 2005-2015 and 2010-2017, respectively. Study visits occurred at baseline and every 6 months thereafter, as needed per routine care. Effectiveness was assessed with changes in ASDAS, BASDAI, BASFI, MDGA, HAQ-DI, PtGA, back pain and acute phase reactants. Safety was evaluated with the incidence of adverse events (AEs) and drug survival rates.

Results: Of the 389 IFX- and 421 GLM-treated patients, the proportion of males were $62.7 \%$ and $59.1 \%$, the mean age were 45.6 and 45.7 years and the mean disease duration were 8.6 and 6.0 years, respectively. Most patients were bio-naive $(>82.7 \%)$. Interestingly, we observed a significant decrease in disease duration in the IFX cohort from a median of 8.0 to 3.5 and 1.0 years in 2005-2008, 2009-2012 and 2013-2015, respectively $(p<0.001)$. A reduction in baseline BASFI score $(6.3$ vs. 5.9 vs $5.1 ; P=0.011)$ and in the proportion of patient in ASDAS very high disease activity $(48.4 \%, 43.8 \%, 30.3 \% ; \mathrm{p}=0.004)$ were also observed. As for the GLM cohort, most disease parameters including median disease duration (1.6 years), mean baseline BASFI (5.3) and the proportion of patients in ASDAS very high disease activity $(48 \%)$ remained similar from 2010-2017.

Treatment with both IFX and GLM significantly improved all disease parameters over time $(\mathrm{P}<0.001)$ from baseline up to 120 and 84 months, respectively, with similar efficacy between agents.

AEs were reported for $67.9 \%$ and $70.5 \%$ (136 and 131 events/100 PYs) and SAEs for $15.4 \%$ and $8.1 \%$ (10.5 and 22.7 events/100 PYs) covering 1251.3 and 674.8 years of exposure for IFX- and GLM-treated patients, respectively. The most frequently occurring $\mathrm{AEs}$ ( $>7 \%$ of patient in either group) were upper respiratory tract infection, arthralgia and back pain Two deaths occurred in IFX-treated patients (myocardial infarct, drowning) and two among GLM-treated patients (oropharyngeal cancer; neutropenia staphylococcal/pseudomonas infections, septic shock).

The proportion of patients who discontinued treatment were $65.8 \%$ over a mean 3.2 years of exposure in the IFX cohort and $56.8 \%$ over 1.6 years in the GLM cohort

Conclusion: Both IFX and GLM treatment significantly reduced disease activity and improved functionality in a similar fashion and were well tolerated in patients with AS. Differences in baseline characteristics over time demonstrate improvement in early diagnosis of AS and earlier access to biologic therapies.
Disclosure of Interests: Proton Rahman: None declared, Derek Haaland Grant/research support from: Janssen Sponsored Study, Dalton Sholter Grant/research support from: Janssen Sponsored Study, Michael Starr: None declared, Arthur Karasik: None declared, Michelle Teo Grant research support from: Janssen Sponsored Study, Sanjay Dixit Grant/ research support from: Janssen Sponsored Study, Consultant for: Janssen, Speakers bureau: Janssen, Ariel Masetto Grant/research support from: Amgen, Sanofi, Consultant for: Sanofi, Pfizer, Bristol-Myers Squibb, Novartis, Boehringer Ingelheim, Speakers bureau: Novartis, Anna Jarosynska Grant/research support from: Janssen Sponsored Study, Pauline Boulos Grant/research support from: Janssen Sponsored Study, Maqbool Sheriff Grant/research support from: Janssen Sponsored Study, Jacqueline Stewart Consultant for: Pfizer, Abbvie, Amgen, Celgene, Roche, Novartis, Merck, Emmanouil Rampakakis : None declared, Odalis Asin Mlilan Employee of: Employee of Janssen, Allen Lehman Employee of: Employee of Janssen, Meagan Rachich Shareholder of: Janssen, Employee of: Employee of Janssen, Francois Nantel Shareholder of: Janssen, Employee of: Employee of Janssen

DOI: 10.1136/annrheumdis-2019-eular.1431

\section{AB0705 AQUILA STUDY IN GERMANY - REAL WORLD ADHERENCE AND PERSISTENCE OF SECUKINUMAB TREATMENT IN ANKYLOSING SPONDYLITIS AND PSORIATIC ARTHRITIS PATIENTS - AN INTERIM ANALYSIS}

Uta Kiltz ${ }^{1}$, Daniel Peterlik ${ }^{2}$, Veronika Winkelmann ${ }^{2}$, Hans-Peter Tony ${ }^{3}$, on behalf of the AQUILA Study Group. ${ }^{1}$ Rheumazentrum Ruhrgebiet, Herne, and Ruhr University, Bochum, Germany; ${ }^{2}$ Novartis Pharma GmbH, Clinical Research Immunology, Hepatology and Dermatology, Nürnberg, Germany; ${ }^{3}$ Medizinische Klinik II, Universitätsklinik, Rheumatology/Immunology, Würzburg, Germany

Background: Secukinumab (SEC) has been shown to be an effective treatment for patients (pts) with ankylosing spondylitis (AS) and psoriatic arthritis (PsA) in several phase III studies ${ }^{1,2}$. Clinical studies reduce confounding factors, but do not guarantee the same results in real world where treating physicians deal with comorbidities, adherence and persistence challenges. Especially medication adherence has a direct impact on health outcomes. However, there is limited information on the adherence and persistence of SEC in routine care.

Objectives: The aim of this interim analysis is to report baseline (BL) characteristics and to assess adherence rate as well as treatment persis tence of SEC in AS and PSA pts under real world conditions.

Methods: AQUILA is an ongoing, non-interventional study enrolling 2000 pts with active AS or PsA in Germany. Adherence was calculated as the proportion of the accumulated actual dose divided by the accumulated target dose (according to summary of product characteristics) during the maintenance phase (week 6 to 52). Adherence rate was calculated as the proportion of pts who achieved an adherence of $>80 \%$ at wk 52 These calculations included pts who discontinued treatment with SEC; still ongoing pts were excluded from this interim analysis. Treatment persistence (time from study inclusion, i.e. date of informed consent, until treatment discontinuation) stratified by biologic/biosimilar (Bx) pretreatment was analyzed using Kaplan-Meier plots.

Results: This interim analysis describes 952 pts (AS $n=311$, PsA $n=641$ ) who were included at BL. In total, $51.4 \%(\mathrm{n}=489)$ of the pts were female and $48.6 \%(n=463)$ male, mean age was 50.8 years, and $67.8 \%(n=645)$ were pretreated with Bx. At treatment start with SEC, pts presented with a number of extra-articular manifestations/comorbidities: plaque psoriasis $11.6 \%(n=36)$ in AS and $66.3 \%(n=425)$ in PsA, uveitis $6.4 \%(n=20)$ in AS and $1.7 \%(n=11)$ in PsA, depression $15.4 \% \quad(n=48)$ in AS and $15.4 \%$ $(n=99)$ in PsA, coronary heart disease $3.5 \% \quad(n=11)$ in AS and $7.9 \%$ $(n=51)$ in PsA, stroke $0.6 \%(n=2)$ in $A S$ and $1.9 \% \quad(n=12)$ in PsA, and heart insufficiency $1.6 \%(n=5)$ in AS and $3.0 \%(n=19)$ in PsA.

Adherence rates for SEC $[95 \% \mathrm{Cl}]$ at week 52 were $64.5 \%[58.3,70.5$ and $56.0 \%[51.5 ; 60.4]$ for AS and PsA, respectively.

In both $\mathrm{AS}$ and $\mathrm{PsA}$, more Bx-pretreated pts discontinued treatment than Bx-naïve pts (AS: $38.0 \%$ versus $18.9 \%$; PsA: $32.3 \%$ versus $20.7 \%$ ). The most frequent reason for discontinuation was "adverse event/insufficient response/loss of efficacy" in both Bx-pretreated ( $n=73, \quad 33.0 \% / n=111$, $26.2 \%)$ as well as in Bx-naïve ( $n=10,11.1 \% / n=34,15.7 \%)$ AS/PsA pts. Moreover, Kaplan-Meier analysis showed that in both AS (Figure 1a) and PsA (Figure 1b) groups, Bx-naïve pts who received SEC as first line therapy had a higher persistence rate than those pretreated with Bx. 